\title{
Numerical renormalization group study of two-channel three-impurity triangular clusters
}

\author{
Rok Žitko ${ }^{1,2}$ and Janez Bonča ${ }^{3,2}$ \\ ${ }^{1}$ Institute for Theoretical Physics, University of Göttingen, Friedrich-Hund-Platz 1, Göttingen, Germany \\ ${ }^{2}$ J. Stefan Institute, Ljubljana, Slovenia \\ ${ }^{3}$ Faculty of Mathematics and Physics, University of Ljubljana, Ljubljana, Slovenia
}

(Dated: November 21, 2018)

\begin{abstract}
We study triangular clusters of three spin- $1 / 2$ Kondo or Anderson impurities that are coupled to two conduction leads. In the case of Kondo impurities, the model takes the form of an antiferromagnetic Heisenberg ring with Kondo-like exchange coupling to continuum electrons. We show that this model exhibits many types of the behavior found in various simpler one and two-impurity models, thereby enabling the study of crossovers between a number of Fermi-liquid (FL) and non-Fermi-liquid (NFL) fixed points. In particular, we explore a direct crossover between the two-impurity Kondo-model NFL fixed point and the two-channel Kondo-model NFL fixed point. We show that the concept of the two-stage Kondo effect applies even in the case when the first-stage Kondo state is of NFL type. In the case of Anderson impurities, we consider the transport properties of three coupled quantum dots. This class of models includes as limiting cases the familiar serial double quantum dot and triple quantum dot nanostructures. By extracting the quasiparticle scattering phase shifts, we compute the low-temperature conductance as a function of the inter-impurity tunneling-coupling. We point out that due to the existence of exponentially low temperature scales, there is a parameter range where the stable "zero-temperature" fixed point is essentially never reached (not even in numerical renormalization group calculations). The "zero-temperature" conductance is then of no interest and it may only be meaningful to compute the conductance at finite temperature. This illustrates the perils of studying the conductance in the ground state and considering thermal fluctuations only as a small correction.
\end{abstract}

PACS numbers: 75.30.Hx, 71.10.Hf, 72.10.Fk, 72.15.Qm

\section{INTRODUCTION}

Quantum impurity models describe localized single impurities or impurity clusters in interaction with conduction bands of itinerant electrons. They appear in several different contexts in condensed matter physics: as models for dilute magnetic impurities in metals $1,2,3$, as models of semiconductor quantum dots and other nanostructures embedded between conduction leads ${ }^{4.5}$ and as effective models within the dynamical mean-field theory of bulk correlated electron systems ${ }^{6}$. Quantum impurity models are often studied also for their own sake due to the fascinating and rich behavior that they exhibit. Advances in the computational resources and improved implementations of the numerical renormalization group (NRG) technique $7,8,9,10,11,12,13,14,15,16,17,18,19,20$ make possible accurate and detailed studies of increasingly complex quantum impurity models featuring several impurities and several conduction channels ${ }^{21,22,23,24,25,26,27,28,29}$.

NRG permits to calculate finite-size excitation spectra, thermodynamic properties (such as impurity contributions to the magnetic susceptibility and entropy), various correlation functions (in particular zero-frequency correlators) and conductance through nanostructures both at zero temperature and at finite temperatures ${ }^{20}$. Such detailed knowledge about the behavior of the system under study at different temperature scales can be used to establish phase diagrams which delineate the parameter ranges with characteristic properties. In more technical terms, NRG allows to determine the possible fixed points of the model, their stability with respect to various perturbation and crossovers between the fixed points. Depending on the nature of the excitations, the fixed points may be classified as either Fermi-liquid (FL) or non-Fermi- liquid (NFL) fixed points ${ }^{30,31,32}$. The excitation spectra of FL fixed points can be mapped one-to-one to the spectra of free non-interacting fermions (electrons); they have a characteristic appearance of equally-spaced lowest lying excited states. The excitation spectra of NFL fixed points cannot be related to non-interacting fermionic systems, they are typically more complex and the lowest lying excited states are not equally spaced (in some cases, however, the spectra may be described in terms of real Majorana fermions with twisted boundary conditions; the excitation spectra may then be given in terms of fractions ${ }^{33,34,35,36,37,38}$ ).

Non-Fermi-liquid properties of strongly correlated materials and proposed corresponding theoretical models have attracted the interest of the condensed matter community due to the very uncommon situation where the behavior of the system is radically different from what might be expected from the nature of its elementary constituents. The simplest models where NFL behavior emerges are quantum impurity models such as the two-channel single spin- $1 / 2 \mathrm{impu}-$ rity Kondo mode $\mathrm{l}^{30,39,40}$. In this model, the conduction band electrons attempt to screen the impurity moment as in the conventional Kondo effect, but as there are two conduction channels they tend to overscreen a single spin- $1 / 2$ impurity. Since a strong-coupling state with overscreening is not stable the system ends up, instead, in a non-trivial intermediatecoupling NFL state ${ }^{30}$. A mesoscopic system which exhibits the two-channel Kondo effect has recently been experimentally demonstrated $\stackrel{41}{ }$.

In this work, we apply the numerical renormalization group techniques to study a complex impurity model which consists of three spin-1/2 Kondo-model-like impurities coupled by exchange interaction so as to form a Heisenberg ring. Two of the 
impurities are furthermore coupled to two conduction bands. This system is particularly interesting in that it has a rich phase diagram which includes fixed points known from simpler quantum impurity models. In contrast to simpler models, however, the three-impurity model allows to study crossovers between various non-trivial fixed points. Spurred on by a number of experimental achievements ${ }^{42.43,44,45,46,47,48,49}$, the interest in the transport properties of three-impurity models recently intensified $d^{50,51,52,53,54,55,56,57,58,59,60,61,62,63,64,65,66}$. We thus also study the conductance through a system of three coupled quantum dots described by a related model featuring three Anderson-model-like impurity electron levels interconnected by tunneling coupling and connected to conduction bands by hybridization.

In Section $\amalg$ we present the model and briefly discuss properties of known limiting cases. In Section $\amalg$ we discuss the fixed points that are expected to characterize the various parameter ranges. Numerical results for the Kondo-like model are presented in Section IV] In this section we demonstrate the possibility of a direct cross-over between the two-impurity Kondo model fixed point to the two-channel Kondo model fixed point. At the same time, this result also establishes the validity of the two-stage Kondo screening concept in the case where the first stage of screening results in a non-Fermi-liquid fixed point. In Section $\nabla$ we discuss the transport through a triangular triple quantum dot systems connected to two conduction leads. We emphasize that the notion of the "zerotemperature" conductance is of limited utility in systems with exponentially low energy scales, since experiments are performed at finite temperatures.

\section{MODEL}

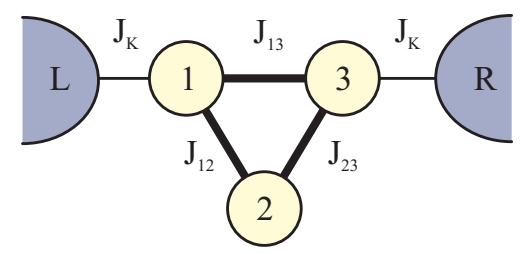

Figure 1: (Color online) Two-channel three-impurity triangular cluster.

The three-impurity Kondo model with two conduction channels, represented schematically in Fig. 1, is described by the Hamiltonian $H=H_{\mathrm{b}}+H_{\text {imp }}+H_{\mathrm{c}}$, where

$$
\begin{aligned}
H_{\mathrm{b}} & =\sum_{\nu \in\{L, R\}, k, \sigma \in\{\uparrow, \downarrow\}} \epsilon_{k} c_{\nu k \sigma}^{\dagger} c_{\nu k \sigma}, \\
H_{\mathrm{imp}} & =\sum_{i<j \in\{1,2,3\}} J_{i j} \boldsymbol{S}_{i} \cdot \boldsymbol{S}_{j}, \\
H_{\mathrm{c}} & =J_{K}\left(\boldsymbol{S}_{1} \cdot \boldsymbol{s}_{L}+\boldsymbol{S}_{3} \cdot \boldsymbol{s}_{R}\right) .
\end{aligned}
$$

The two $(\nu=L$ and $\nu=R)$ conduction bands are assumed to have linear dispersion, $\epsilon_{k}=D k$, where the dimensionless wave-number $k$ ranges from -1 to 1 so that the bandwidth is
$2 D$. The impurities are described by the spin- $1 / 2$ operators $\boldsymbol{S}_{i}$ with $i=1,2,3$ and $\boldsymbol{s}_{\nu}$ is the conduction-band spin density at the position of impurity 1 (3) for $\nu=L(R)$,

$$
\boldsymbol{s}_{\nu}=\sum_{\alpha \alpha^{\prime}}\left(\frac{1}{\sqrt{N}} \sum_{k} c_{\nu k \alpha}^{\dagger}\right)\left(\frac{1}{2} \boldsymbol{\sigma}_{\alpha \alpha^{\prime}}\right)\left(\frac{1}{\sqrt{N}} \sum_{k^{\prime}} c_{\nu k^{\prime} \alpha^{\prime}}\right),
$$

where $N$ is the number of conduction-band states, $c_{\nu k \alpha}^{\dagger}$ and $c_{\nu k \alpha}$ are creation and annihilation operators for electron in band $\nu$ with spin $\alpha \in\{\uparrow, \downarrow\}$, and $\sigma_{\alpha \alpha^{\prime}}$ is the vector of Pauli matrices $\left(\sigma_{\alpha \alpha^{\prime}}^{x}, \sigma_{\alpha \alpha^{\prime}}^{y}, \sigma_{\alpha \alpha^{\prime}}^{z}\right) . \quad J_{K}$ is the antiferromagnetic Kondo exchange constant. Finally, $J_{i j}$ parameterize the inter-impurity exchange interaction. We reduce the parameter space by considering only models with left-right mirror symmetry (parity), i.e. $J_{12}=J_{23}$. While the parity-breaking is a relevant perturbation at some of the fixed points ${ }^{67}$, its effect will not be studied in much detail in this work. We parameterize the exchange constants by

$$
\begin{aligned}
J_{13} & =J_{0} \sin (\beta \pi / 2), \\
J_{12}=J_{23} & =J_{0} \cos (\beta \pi / 2) .
\end{aligned}
$$

Parameter $\beta$ thus parameterizes the asymmetry (ratio) between the exchange coupling between the impurities 1 and 3 in the upper arm of the triangle and the exchange coupling between the side-coupled impurity 2 and the impurity 1 (or 3 ). The special cases are:

- $\beta=1$, which corresponds to the two-impurity Kondo model (plus one totally decoupled impurity);

- $\beta=0$, which corresponds to a linear chain of three impurities;

- $\beta=1 / 2$ which corresponds to a symmetric antiferromagnetic Heisenberg ring, which is a magnetically frustrated system with two degenerate doublets in the ground state.

Parameter $J_{0}$ sets the overall scale of the inter-impurity exchange coupling.

In the two-impurity Kondo mode1 68 (i.e. $\beta=1$ limit), there is a double Kondo screening regime for low inter-impurity exchange interaction $J=J_{0}$ and an inter-impurity-singlet

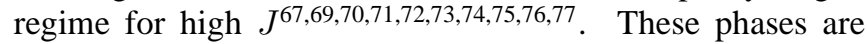
separated by a quantum phase transition ${ }^{78,79}$ at $J=J_{2 \mathrm{KK}}^{*}=$ $c T_{K}^{(1)}$ where $T_{K}^{(1)}$ is the Kondo temperature for a system of a single impurity coupled to a single conduction channel with the same $J_{K}$ (the proportionality constant $c$ is of order 1; often quoted value is $\sim 2.2$ obtained in the early NRG studies ${ }^{70}$, however this particular value is not universal and true $c$ depends on the details of the model and on the chosen definition of the Kondo temperature). Exactly at the transition point, the system has a non-Fermi-liquid ground state and exhibits quantum criticality. This state is, however, unstable and for $J-J_{2 \mathrm{IK}}^{*} \neq 0$ the systems flows to either of the two possible FL fixed points. We denote this NFL fixed point by 2IK. Recently, the two-impurity models were reexamined and it was shown that this fixed point is robust with respect 
to parity, particle-hole symmetry breaking and various other asymmetries $^{74}$.

The system of three impurities in series (i.e. $\beta=0$ limit) has been studied in Ref. 80. This system has a non-Fermiliquid ground state of the same type as the two-channel Kondo model $(2 \mathrm{CK}) 34,40,50,67,81,82,83,84,85,86,87,88,89,90,91$. For low interimpurity exchange interaction $J$, the local moment screening occurs in two stages: at the higher Kondo temperature $T_{K}^{(1)}$ the local moments on impurities 1 and 3 are screened, while the local moment on impurity 2 is screened at an exponentially reduced second Kondo temperature $T_{K}^{(2) 80,92}$. For high $J$, the three spins first lock into an antiferromagnetic spin-chain at $T \sim J$ and the collective spin- $1 / 2$ undergoes Kondo screening at some lower temperature $T_{2 \mathrm{CK}}$ which depends nonmonotonically on $J^{80,93}$. The low-temperature two-channel Kondo (2CK) fixed point is stable with respect to particle-hole symmetry breaking ${ }^{94}$, but it is unstable with respect to parity breaking 40,67,80,95.

For $\beta=0.5$ and sufficiently large $J_{0}$, the three impurities behave as a frustrated antiferromagnet with two degenerate ground state doublets at temperatures on the scale of $J_{0}$. The symmetry is broken by the coupling to the leads as there are only two conduction channels. It should be noted that in the more symmetric case of three conduction channels frustration induces a new type of non-Fermi-liquid behavior ${ }^{23,24}$.

\section{EXPECTED REGIMES}

The behavior of the system at various temperatures and inter-impurity coupling strengths is governed by the proximity to one of the following fixed points (see schematic representations in Fig. 2):

a) three independent local moments, LM,

b) inter-impurity singlet (plus a decoupled spin-1/2 local moment), $\mathrm{S}$,

c) Kondo screening with $\pi / 2$ phase shifts (plus a decoupled spin-1/2 local moment), DK,

d) two-impurity Kondo model non-Fermi-liquid fixed point (plus a decoupled spin-1/2 local moment), 2IK,

e) frustrated antiferromagnetic Heisenberg ring, FR,

f) antiferromagnetic spin chain with $S=1 / 2$, AFM (two different fixed points exist, depending on $J_{12}>J_{13}$ or $\left.J_{12}<J_{13}\right)$

g) two-channel spin-1/2 Kondo model non-Fermi-liquid fixed point, $2 \mathrm{CK}$.

It should be noted that we have restrained ourselves to the fixed points which occur in the mirror (parity) and particlehole symmetric case. In generic model, some of these fixed points are extended into lines or planes of fixed points. In particular, there appears a plane of Fermi-liquid fixed points (plus a decoupled spin-1/2 local moment) parameterized by two continuous quantities (phase shifts in even and odd scattering channel), of which the fixed points $S$ and DK are special cases. There is furthermore a new plane of Fermi-liquid fixed points that is also parameterized by the two phase shifts but this time there is no decoupled local moment. The first plane (P1) occurs for $\beta=1$ and the second one (P2) for $\beta \neq 1$; for $\beta \lesssim 1$, the crossover from $\mathrm{P} 1$ to $\mathrm{P} 2$ occurs by a Kondo-like screening of the nearly decoupled local moment.
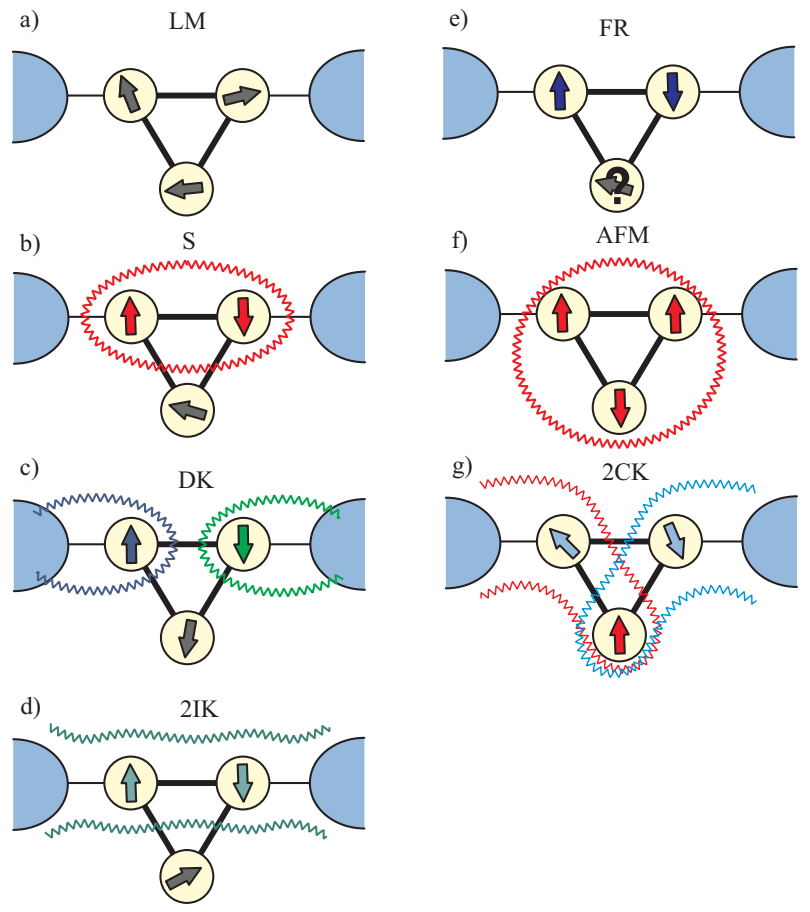

Figure 2: (Color online) Schematic representations of the possible fixed points.

In Fig. 3 we present a schematic "phase diagram" of the system in the $\left(J_{12}, J_{13}\right)$ [or, equivalently, $\left(J_{0}, \beta\right)$ ] plane at very low $(T \rightarrow 0)$ temperatures. Along the $\beta=1$ line we find the $S$ and DK Fermi-liquid fixed points, separated by the 2IK quantum phase transition. Along the $\beta=0$ line as well as in the rest of the plane, the system always ends up in the $2 \mathrm{CK}$ fixed point. This is a special property of the parity-symmetric case (in the fully generic case, only P1 and P2 fixed points are stable). For $T>\max \left(J_{12}, J_{13}\right)$, the system is in the local moment LM regime, while for $T \lesssim \min \left(J_{12}, J_{13}\right)$ the system is described by FR or one of the two AFM fixed points, depending on the values of $J_{12}$ and $J_{13}$. In the region of the parameter plane in close vicinity of the $2 \mathrm{IK}$ fixed point, i.e. for $J_{0} \sim J_{2 \mathrm{IK}}^{*}$ and $\beta \sim 1$, the system first approaches the $2 \mathrm{IK}$ fixed point, then crosses over into the stable $2 \mathrm{CK}$ fixed point.

We emphasize that the 2CK fixed point dominates the lowtemperature phase diagram, i.e. it is the stable fixed point for all $\beta \neq 1$. The values of parameters $J_{0}$ and $\beta$ affect only the way in which this fixed point is approached. A similar reasoning as in the $\beta=0$ case $^{80}$ can also be applied to the general model. At some low enough energy scale, the impurity cluster and the nearby conduction band electrons effectively form a spin- $1 / 2$ object. This object is very local- 
ized in the large- $J_{0}$ limit (when the three impurities align antiferromagnetically into a spin- $1 / 2$ state) or extended in the small- $J_{0}$ limit (in which case the composite spin- $1 / 2$ object is formed by the spin-1/2 of the impurity 2 and two collective spin-singlet states each consisting of an impurity and electrons which screen its spin). In both cases the coupling of this spin$1 / 2$ object to the rest of the system (conduction band electrons at still lower energies in each of the lead) is antiferromagnetic; for large $J_{0}$ this is explicit, since $J_{K}$-terms in the Hamiltonian describe an AFM exchange interaction, while for small $J_{0}$ this is expected by analogy with the two-stage Kondo effect in side-coupled geometry $18,101,102$. Thus the effective model takes the form of a single-impurity two-channel Kondo model. Since we consider reflection-symmetric models in this work, the exchange coupling to both channels are the same, thus the effective model with flow to the stable 2CK NFL fixed point. Of course, in the absence of reflection symmetry, the system would eventually end up in a Fermi-liquid fixed point (in the P2 plane).

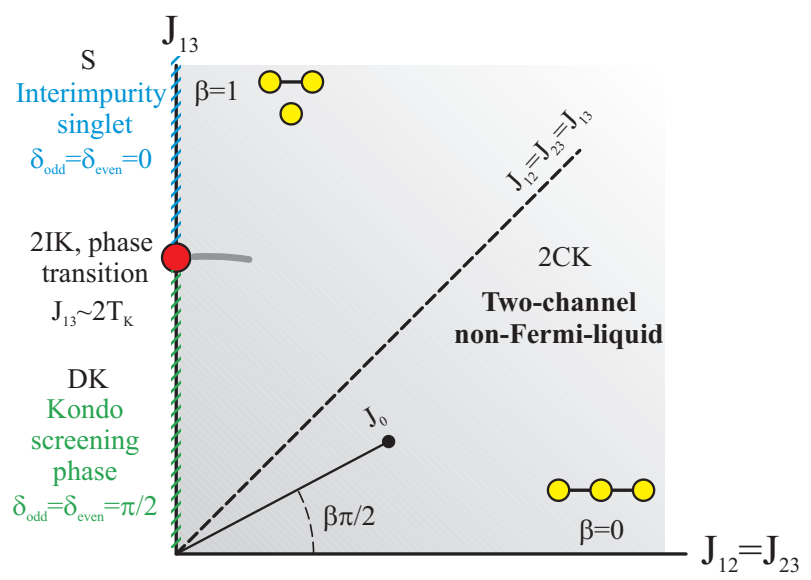

Figure 3: (Color online) Schematic phase diagram of the triangular three impurity Kondo model at low temperatures.

The presence of all the enumerated fixed points and the proposed features of the phase diagram are fully supported by the results of the numerical renormalization group calculation which are detailed in the following sections.

\section{NUMERICAL RESULTS}

Calculations have been performed using the "NRG Ljubljana" package 96 . The impurity model, Eq. (1), is particle-hole symmetric; in fact, it has a larger $\mathrm{SU}(2)$ isospin symmetry of which the particle-hole transformation symmetry is merely a subgroup $25,67,70$. We performed all calculations taking explicitly into account spin $\mathrm{SU}(2)$, isospin $\mathrm{SU}(2)$ and mirror $\mathrm{Z}_{2}$ symmetry groups $7,12,20,25,97,98,99$. We have used the discretization scheme described in Ref. 99 with the discretization parameter $\Lambda=4$. Averaging over four values of the twist parameter $z$ has been used ${ }^{10}$. The NRG truncation cutoff was set at the cutoff energy of $E_{\text {cutoff }}=9 \omega_{N}$, where $\omega_{N}$ is the characteristic energy scale at the $N$-th NRG iteration, or at most 4000 states (which corresponds to approximately 32000 states taking into account the degeneracies). To prevent systematic errors, care is taken not to truncate within a cluster of nearby almost degenerate states. In all calculations presented in this article, we have used $\rho J_{K}=0.2$ which corresponds to the Kondo temperature $T_{K}^{(1)} \approx 0.003 D$ in the single-impurity Kondo model with the same parameter $J_{K}$. (Here we use Wilson's definition of the Kondo temperature, i.e. for $S=1 / 2$ Kondo model one has $k_{B} T_{K} \chi\left(T_{K}\right) /\left(g \mu_{B}\right)^{2}=0.701$ or $k_{B} T_{K} \chi(0) /\left(g \mu_{B}\right)^{2}=0.103$. This definition is commonly, albeit not exclusively, used in NRG literature.)

We first ascertain the presence of the expected fixed points by calculating the finite-size excitation spectra. These spectra can be represented in the form of the "renormalization flow diagrams", some of which are shown in Fig. 4. Flow diagrams show the NRG eigenvalue spectrum in units of the characteristic energy (or, equivalently, temperature) scale $\omega_{N} \propto \Lambda^{-N / 2}$ as a function of the NRG iteration number $N$ (see Refs. 7, 12 and 20). We join the points by lines for easier visualization and interpretation. The colors (shades of gray) correspond to different sets of quantum numbers for the total isospin $(I)$, total spin $(S)$ and parity $(P)$. The system is said to be near some fixed point when the eigenvalues do not change much between successive iterations (i.e. the lines are horizontal), while crossovers correspond to transitions between such regions.

For $\Lambda=4$, the single-particle eigenvalues which can be combined to give the Fermi liquid fixed point eigenspectra of a single Wilson chain with no impurities are ${ }^{7,12}$

$$
\begin{aligned}
& \eta_{j}^{*}=0.8589029,3.99452, \Lambda^{2}, \Lambda^{3}, \ldots, \quad N \text { odd } \\
& \hat{\eta}_{j}^{*}=1.983281,7.999996, \Lambda^{5 / 2}, \Lambda^{7 / 2}, \ldots, \quad N \text { even },
\end{aligned}
$$

for odd and even iteration number $N$, respectively. In the first row of Fig. 4 we show the NRG eigenvalue flow for $\beta=1$ for three values of $J_{0}$. For $J_{0} / D=0.0154$, we have $J_{0}<J_{2 \mathrm{IK}}^{*}$ and we expect a flow to the Fermi-liquid double-Kondo (DK) fixed point with $\delta_{\mathrm{q} \cdot \mathrm{p} \text {. }}^{\text {even }}=\delta_{\mathrm{q} . \mathrm{p} \text {. }}^{\text {odd }}=\pi / 2$ quasiparticle scattering phase shifts. The $\pi / 2$ phase shifts in both channels imply that the excitation spectrum of even-length Wilson chains correspond to that of odd-length non-interacting chain. Indeed, the lowest excitation energies are $\eta_{0}^{*}, 2 \eta_{0}^{*}, 3 \eta_{0}^{*}, \ldots$ to high accuracy. The quantum numbers are, however, different from those of the related two-impurity problem due to the presence of a decoupled spin- $1 / 2$ impurity (which also implies an additional two-fold degeneracy of all levels). For $J_{0} / D=0.0159$, we have $J_{0}>J_{2 \mathrm{IK}}^{*}$, which corresponds to the flow to the Fermi-liquid inter-impurity singlet (S) phase with $\delta_{\mathrm{q} . \mathrm{p} \text {. }}^{\text {even }}=\delta_{\mathrm{q} . \mathrm{p} \text {. }}^{\text {odd }}=0$ phase shifts. The excitation spectrum of even-length Wilson chains corresponds to that of even-length non-interacting chain with lowest excitation energy $\hat{\eta}_{0}^{*}$. Finally, the unstable fixed point for $N=10, \ldots, 20$ at $J_{0} \sim J_{2 \mathrm{IK}}^{*} \approx 0.0158 D$ is the $2 \mathrm{IK}$ NFL fixed point with the energy spectrum (after suitable rescaling) described by the fractions $3 / 8,1 / 2,7 / 8,1, \ldots 33,67$.

The second row in Fig. 4 shows the flow diagrams for constant $\beta=0.92$ for a range of values of $J_{0}$. In all cases the system ends up in the same stable fixed point, which is the $2 \mathrm{CK}$ 


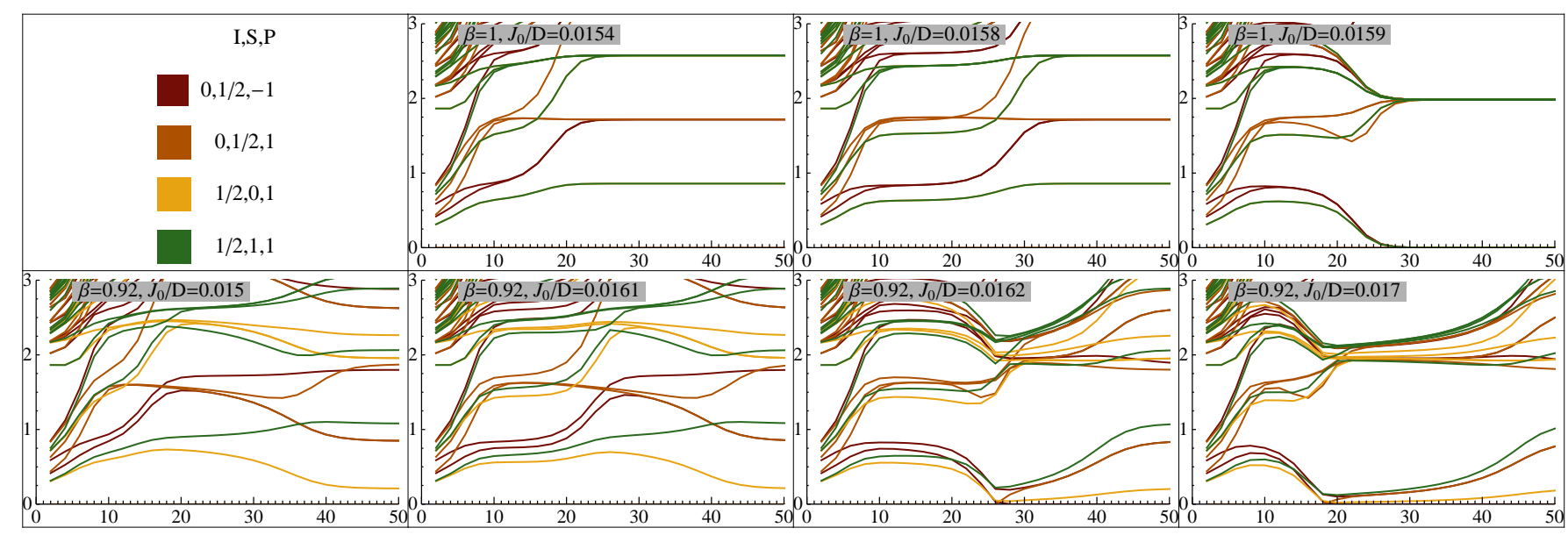

Figure 4: (Color online) NRG eigenvalue renormalization flow diagrams for even iteration numbers $N$. The states are indexed by the total isospin, total spin and parity quantum numbers.

NFL fixed point with energy spectrum (after suitable rescaling) $1 / 8,1 / 2,5 / 8,1,1+1 / 8, \ldots$, as predicted by the boundary conformal field theory approach to the $2 \mathrm{CK}$ problem $\$ 1$. Note the similarities in the flow diagrams in the first and second line, especially at high and intermediate temperatures (up to $N \sim 20$ ). At lower temperatures, the coupling to the impurity 2 eventually drives the system to the $2 \mathrm{CK}$ fixed point for any value of $\beta \neq 1$ and $J_{0}$.

Basic information about the magnetic correlations within the three impurity cluster may be obtained by considering the spin-spin correlations $c_{i j}=\left\langle\boldsymbol{S}_{i} \cdot \boldsymbol{S}_{j}\right\rangle$ at zero temperature. These provide insight in the competition between the inter-impurity interactions $J_{i j}$ and the impurity-lead Kondo exchange interaction $J_{K}$. In Fig. 5 we plot $c_{13}$ and $c_{12}$ both as a function of the interaction strength $J_{0}$ and as a function of the asymmetry ratio $\beta$. Note that if the impurities were decoupled from the conduction channels (i.e. for $J_{K} / J_{0} \equiv 0$ ), the spin correlation at zero temperature would depend only on the parameter $\beta$, not on $J_{0}$. The behavior of the decoupled cluster approximates the properties of the impurity system in the large $J_{0}$ limit, see the $J_{0} / D=1$ plots in Fig. 5 b. At $\beta=1 / 2$, the decoupled cluster has $C_{3 v}$ symmetry, thus $c_{12}=c_{13}$. The two degenerate spin-doublets at this point are

$$
\begin{aligned}
& \left|a_{\sigma}\right\rangle=1 / \sqrt{2}(|\uparrow, \sigma, \downarrow\rangle-|\downarrow, \sigma, \uparrow\rangle), \\
& \left|b_{\sigma}\right\rangle=1 / \sqrt{6}(|\downarrow, \uparrow, \sigma\rangle+|\uparrow, \downarrow, \sigma\rangle-2|\uparrow, \uparrow, \bar{\sigma}\rangle) .
\end{aligned}
$$

For an equal mixture of these two states, we expect $c_{12}=$ $c_{13}=-1 / 2$. For $\beta \neq 1 / 2$ the degeneracy is lifted. For $\beta>1 / 2$, the decoupled impurities are in state $|a\rangle$, which corresponds to a local singlet state between impurities 1 and $3\left(c_{13}=-3 / 4\right)$, while the impurity 2 is decoupled $\left(c_{12}=0\right)$. For $\beta<1 / 2$, the decoupled impurities are in state $|b\rangle$, which corresponds to a rigid antiferromagnetic spin chain $\left(c_{12}=\right.$ $\left.-1 / 2, c_{13}=1 / 4\right)$.

In the full model $\left(J_{K} \neq 0\right)$, the degeneracy between the doublets is lifted even at $\beta=1 / 2$ by the coupling to the channels. Curiously, in the limit $J_{0} \gg J_{K}$, the correlations $c_{12}$ and $c_{13}$ are not only different, but they even have opposite signs. This is explained by Fig. 5b: the point where $c_{12}=c_{13}$ is shifted from the value of $\beta=1 / 2$. We also note that at large $J_{0}$, the transition between $\left|a_{\sigma}\right\rangle$ and $\left|b_{\sigma}\right\rangle$ impurity ground states becomes increasingly sharp, thus a small change in $\beta$ leads to an abrupt change in the spin correlations. The spinspin correlations curves at $\beta=1 / 2$ play the role of separatrix between two different limiting regimes (dashed curves in Fig. 5 5 . In the other limit of $J_{0} \ll J_{K}$, the spin correlations tend to zero due to the magnetic screening by conduction electrons. It may also be noted that the "side-coupled" impurity 2 is aligned antiferromagnetically with respect to the "directlycoupled" impurities 1 and 3 for any values of parameters $J_{0}$ and $\beta \neq 1$. Since antiferromagnetic exchange is a relevant perturbation, this implies that the local moment on impurity 2 will always be screened (except for $\beta=1$ ).

At $\beta=1$, we have studied $c_{13}=\left\langle\boldsymbol{S}_{1} \cdot \boldsymbol{S}_{3}\right\rangle$ at the 2IK critical point, $J_{0}=J_{2 \mathrm{IK}}^{*}$, which is expected to be equal to $-1 / 4$ due to a degeneracy between one singlet and one triplet state ${ }^{69,100}$. Two general remarks concerning NRG calculations are in order at this point. The first concerns the value of the discretization parameter $\Lambda$. While even very high values of $\Lambda$ typically lead to results which are qualitatively correct (in the single-impurity Anderson model, one can obtain decent magnetic susceptibility curves even at surprisingly high $\Lambda=40$ ), some details depend crucially on taking the $\Lambda \rightarrow 1$ limit. The position of the quantum phase transition as a function of $J$ and the value of $\left\langle\boldsymbol{S}_{1} \cdot \boldsymbol{S}_{3}\right\rangle$ in the two-impurity Kondo model are one such example. At $\Lambda=4$, we find $J_{2 \mathrm{IK}}^{*} / D \approx 0.01585$ and $c_{13} \approx-0.255$, while at $\Lambda=2$ we find $J_{2 \mathrm{IK}}^{*} / D \approx 0.01497$ and $c_{13} \approx-0.253$. The convergence to the expected value of $c_{13}=-1 / 4$ is thus relatively slow. The second remark concerns the averaging over the twist parameter $z$ (the " $z$-trick"). We find that the precise value of the exchange parameter $J$ at the critical point depends slightly on the value of $z$ at constant $\Lambda$, the more so as $\Lambda$ is increased. To accurately study the detailed properties of the model in the vicinity of critical points, the averaging over $z$ is thus better to be avoided and $\Lambda$ should be kept small. Finally, it may also be noted that $c$ 

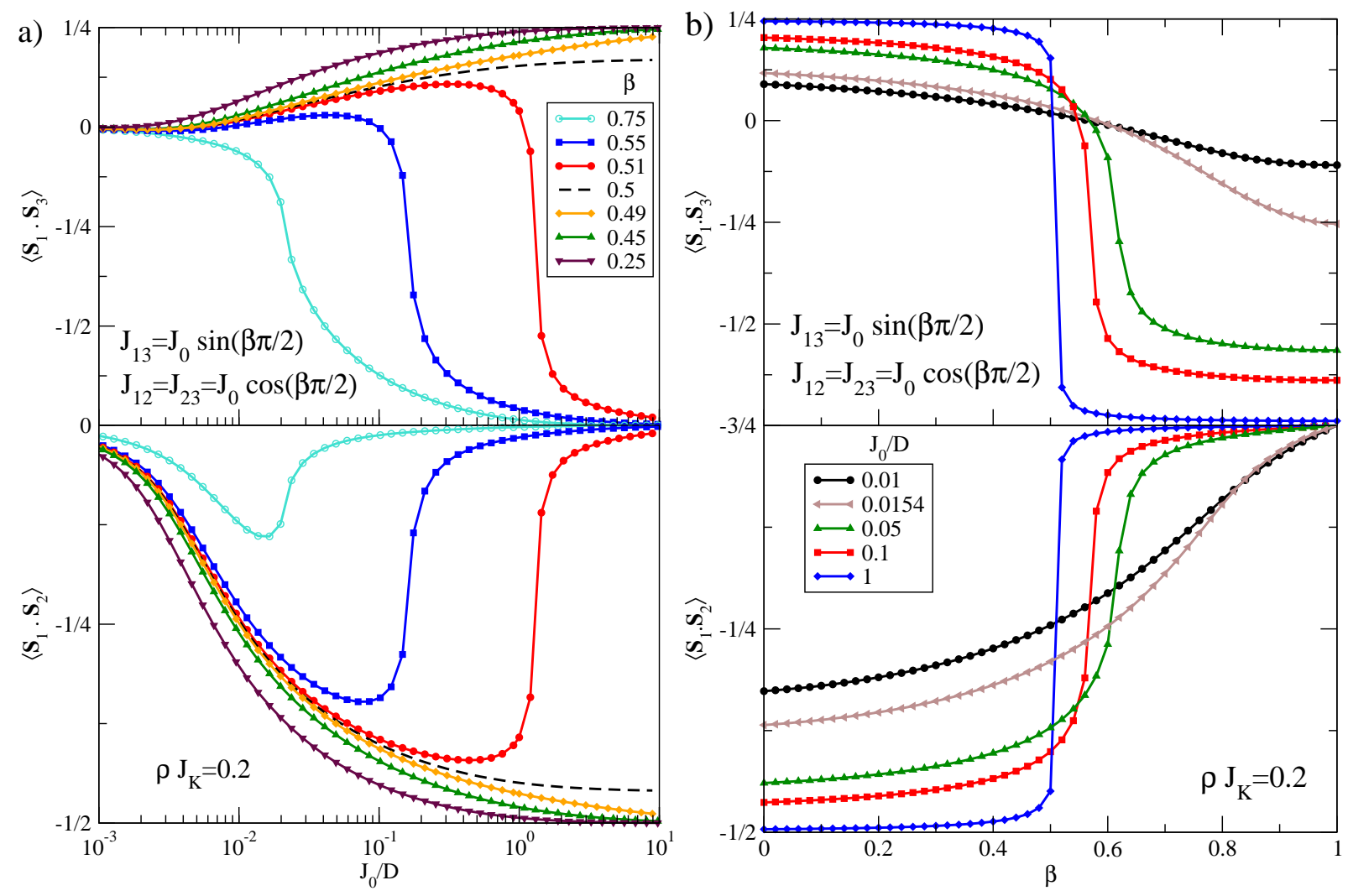

Figure 5: (Color online) Spin-spin correlation functions between the impurities in the upper arm of the triangle $\left(\boldsymbol{S}_{1} \cdot \boldsymbol{S}_{3}\right)$ and between the side-coupled impurity and one of the impurities in the upper arm $\left(\boldsymbol{S}_{1} \cdot \boldsymbol{S}_{2}\right)$. Note that $\boldsymbol{S}_{1} \cdot \boldsymbol{S}_{2}=\boldsymbol{S}_{3} \cdot \boldsymbol{S}_{2}$ due to reflection symmetry. The correlations shown are those at $T=0$; in the major part of the parameter space, the final values are established on the scale $\sim \min \left(J_{13}, J_{12}\right)$ which is typically much higher than the Kondo temperature scales.

in $J_{2 \mathrm{IK}}^{*}=c T_{K}^{(1)}$ is here approximately equal to 5.0 , not 2.2 (still using Wilson's definition of the Kondo temperature), see Section II.

We now focus on the vicinity of the 2IK fixed point, i.e. to the region $\beta \sim 1, J_{0} \sim J_{2 \mathrm{IK}}^{*}$. For $\beta=1$, the system crosses over from LM to 2IK fixed point at the two-impurity Kondo temperature $T_{K}^{(2 \mathrm{IK})}$. Since this fixed point is not stable, the system then crosses over at some lower temperature $T_{\Delta}^{(2 \mathrm{IK})} \propto\left(J-J_{2 \mathrm{IK}}^{*}\right)^{2} / T_{K}^{(2 \mathrm{IK})}$ to either S or DK fixed point, depending on whether $J_{0}>J_{2 \mathrm{IK}}^{*}$ or $J_{0}<J_{2 \mathrm{IK}}^{*}$. This can be observed in Fig. 6 (black curve, $\beta=1$ ), where we plot two thermodynamic quantities, the impurity contribution to the magnetic susceptibility $k_{B} T \chi_{\mathrm{imp}}(T) /\left(g \mu_{B}^{2}\right)=\mu_{\mathrm{eff}}$, and the impurity contribution to the entropy $s_{\text {imp }}(T) / k_{B}=\ln \nu_{\text {eff }}$ as a function of the temperature. Note that the first quantity may be interpreted as the effective impurity cluster magnetic moment $\mu_{\text {eff }}$, while the second one can be related to the effective number of degrees of freedom of the cluster, $\nu_{\text {eff }}$. At the Kondo temperature $T_{K}^{(2 \mathrm{IK})}$, two local moments are screened, while the third decoupled spin remains free; correspondingly, $\mu_{\text {eff }}$ is reduced from $3 / 4$ to $1 / 4$. In the two-impurity Kondo effect, the NFL fixed point is associated with $1 / 2 \ln 2$ residual entropy, thus the entropy goes from the LM value of $3 \ln 2$ to $\ln 2+1 / 2 \ln 2$. The residual entropy of $1 / 2 \ln 2$ is released at
$T_{\Delta}^{(2 \mathrm{IK})}$, when the system crosses over to the FL stable fixed point. Since impurity 2 is completely decoupled at $\beta=1$, there is a residual spin- $1 / 2$ local moment with $\ln 2$ entropy.

Keeping $J_{0}$ constant and decreasing $\beta$, we weakly couple the impurity 2 to the rest of the system, see Fig. 6. One effect is the increased crossover temperature $T_{\Delta}^{(2 \mathrm{IK})}$. More importantly, the local moment on the impurity 2 is now screened at some lower temperature $T_{K}^{2 \mathrm{CK}}$. This can be observed both in $\mu_{\text {eff }}$ curves, where the effective moment is reduced from $1 / 4$ to 0 , and in the effective entropy which is reduced by $1 / 2 \ln 2$, which is a characteristic value for the two-channel Kondo effect. In our mirror-symmetric model, there is no further crossover and the 2CK fixed point is stable. Furthermore, by additional calculations we have checked that $2 \mathrm{CK}$ is the stable fixed point throughout the phase diagram, Fig. 3, for any $J_{0}$ and $\beta \neq 1$, as expected.

By analogy with the two-stage Kondo effect in the case of two impurities that are coupled to a single conduction channel in a side-coupled configuration ${ }^{18,101,102}$, the two-channel Kondo effect due to the side-coupled impurity is expected to occur on a temperature scale that depends exponentially on the effective exchange coupling of this impurity to the other two impurities, $J_{\text {eff }}$, i.e. we expect a function dependence of 


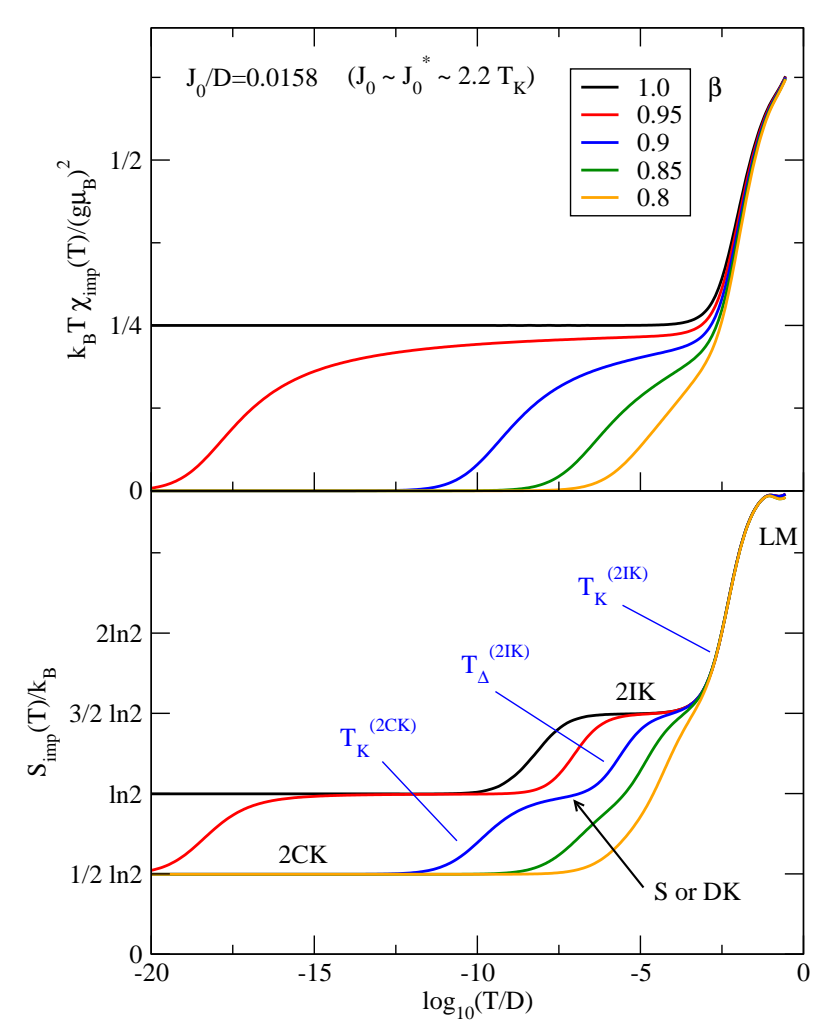

Figure 6: (Color online) Impurity cluster contribution to the magnetic susceptibility and entropy for a range of values of $\beta$ at fixed $J_{0}$.

the form

$$
T_{K}^{(2)}=T_{K}^{(1)} \exp \left(-\frac{1}{\rho_{\mathrm{eff}} J_{\mathrm{eff}}}\right)
$$

where $T_{K}^{(1)}$ and $T_{K}^{(2)}$ are first-stage and second-stage Kondo temperatures, while $\rho_{\text {eff }}$ can be interpreted as the width of the band of the effective local Fermi-liquid quasiparticles resulting from the first-stage Kondo effect, which is proportional to $T_{K}^{(1)}$. In the present situation, $J_{\text {eff }} \propto \cos (\beta \pi / 2)$. From the results of calculations of the magnetic susceptibility along a line of values of $\beta$ (from $\beta=0.9$ to $\beta=0.94$ ) at a fixed value of the exchange coupling $J_{0} / D=0.0158$, we extracted $T_{K}^{2 \mathrm{CK}}$ using the prescription $\mu_{\mathrm{eff}}\left(T_{K}^{2 \mathrm{CK}}\right)=0.07$. Linear regression (see Fig. 7) then gives

$$
\log _{10} \frac{T_{K}^{2 \mathrm{CK}}}{D}=-0.76-1.35 \frac{1}{\cos (\beta \pi / 2)} .
$$

The good agreement confirms our anticipation that Kondo screening in multiple stages can occur whenever additional impurities are (indirectly) weakly coupled to the continuum of electrons via other (directly coupled) impurities. This occurs even in the case of more uncommon types of the Kondo effect.

For $J_{0} / D=0.0158$, the system is already in the Fermiliquid fixed point as the temperature begins to decrease towards the second-stage Kondo screening. We find, however,

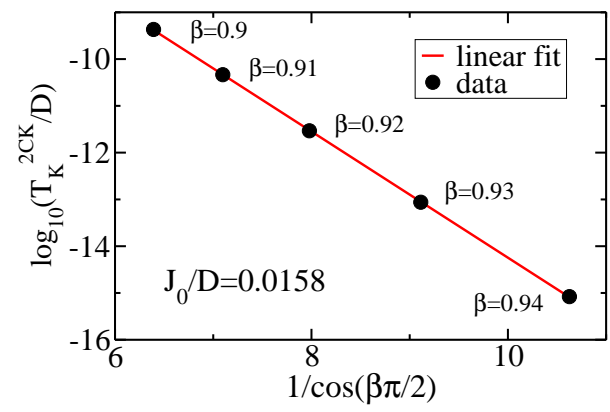

Figure 7: The Kondo cross-over temperature as a function of the parameter $\beta$ for fixed $J_{0} / D=0.0158$.

that the second stage Kondo screening occurs even when after the first stage of the Kondo screening the system cannot be described in terms of the Fermi-liquid quasiparticles, but is rather in a non-Fermi liquid regime. In other words, the continuum of NFL excitations may also serve as an impurity bath in a (second-stage) Kondo effect. By fine-tuning $J_{0}$ around the critical value of $J_{2 \mathrm{IK}}^{*}$ at fixed $\beta=0.95$, we have managed to reduce $T_{\Delta}^{(2 \mathrm{IK})}$ below $T_{K}^{(2 \mathrm{CK})}$, see Fig. 8 . This results in a direct cross-over from $2 \mathrm{IK}$ to $2 \mathrm{CK}$ fixed-point on the temperature scale of $T_{C}$, see the flow diagram in Fig. 9 It appears that the cross-over temperature $T_{C}$ cannot be reduced to lower values by further tuning of the parameter $J_{0}$, which implies that the phase diagram in the $(T, J)$ plane corresponds to that sketched in Fig. 10 The 2IK regime does not extend down to zero temperature as would be the case at $\beta=1$ and there is a single stable $2 \mathrm{CK}$ fixed point at $T=0$. As the temperature is reduced, we can either pass from $2 \mathrm{IK}$ to $2 \mathrm{CK}$ via an intermediate-temperature Fermi-liquid phase DK or S (path a in Fig. 10), or directly from 2IK to 2CK (path b). As $\beta$ is reduced, the region in $(T, J)$ plane that is governed by the $2 \mathrm{IK}$ fixed point, becomes smaller and eventually disappears.

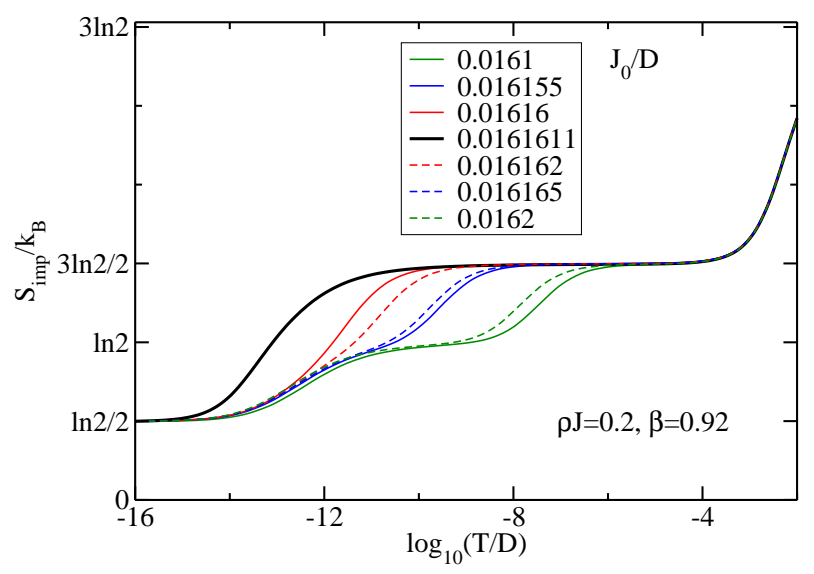

Figure 8: (Color online) Impurity cluster contribution to the entropy for a range of values of $J_{0}$ at fixed $\beta . T_{\Delta}^{2 \mathrm{IK}}$ is driven down until it is made equal to $T_{K}^{2 \mathrm{CK}}$.

It has been shown that in the single-impurity two-channel Kondo model, the Kondo temperature is a non-monotonic function of the Kondo exchange interaction ${ }^{93}$. In the weak- 


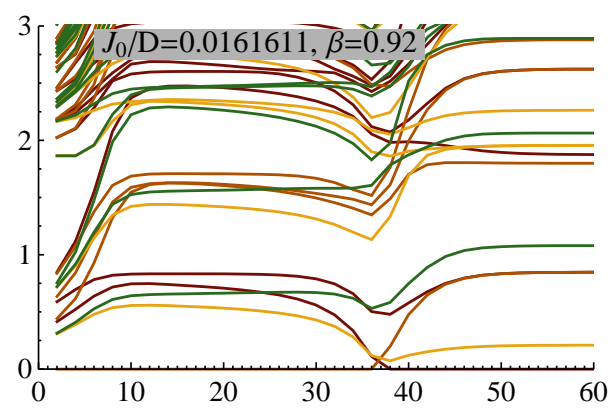

Figure 9: NRG flow diagram in the case of the cross-over from 2IK to $2 \mathrm{CK}$ regimes.

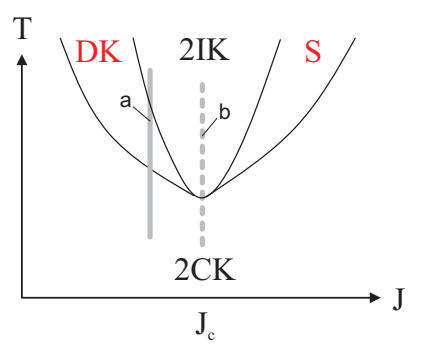

Figure 10: Schematic phase diagram in the $(T, J)$ plane for constant parameter $\beta$.

coupling $g=\rho_{0} J_{K} \ll 1$ regime we have

$$
T_{K}^{(w c)} \approx D e^{-1 / 2 g+\ln (2 g)+O(g)},
$$

while in the strong-coupling $g \gg 1$ regime

$$
T_{K}^{(s c)} \approx D e^{-\gamma g / 2-\ln (\gamma g / 2)+O(1 / h)}
$$

with $\gamma=30 / 46^{93}$. By analogy, we expect that the temperature at which our three impurity system ultimately crosses over to the 2CK fixed point will be a non-monotonous function of $J_{0}$ at any value of $\beta$, with maximum values occurring in the parameter range where $J_{0} \sim T_{K}^{(1)}$ with $T_{K}^{(1)}$ being the Kondo temperature of a single spin- $1 / 2$ Kondo impurity with $\rho J_{K}=0.2$. This is indeed the case. In Fig. 111 we show an overview diagram of the cross-over scale as a function of $\beta$ and $J_{0}$. The numbers displayed are the integer parts of the decadic logarithm of the cross-over temperature. For very small and very large $J_{0}, T_{2 C K}$ is lower than the lowest temperature in the NRG iteration, $\sim 10^{-12} D$. It is worth emphasizing that there is a relatively large section of the parameter space where the cross-over occurs at relatively high temperature. This region is a continuation to the triangular impurity configuration of the intermediate regime found in the linear three-impurity model discussed in Ref. 80 .

We may note in conclusion to this section that the parity breaking destabilizes the $2 \mathrm{CK}$ fixed point, while it is a marginal perturbation for the 2IK fixed point. A small parity breaking of the form $J_{K, L} \neq J_{K, R}$ or $J_{12} \neq J_{23}$ will thus lead to another crossover from the now unstable $2 \mathrm{CK}$ fixed point to a stable FL fixed point (in plane P2) which corresponds to

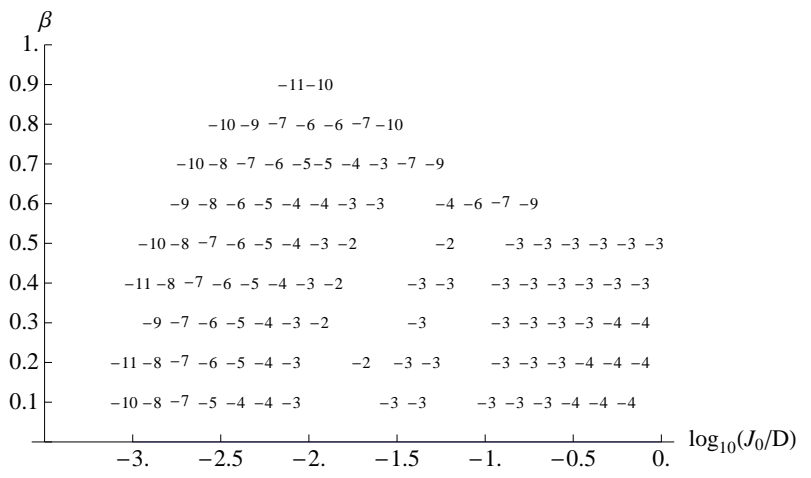

Figure 11: The cross-over (Kondo) temperatures $T_{K}^{(2 \mathrm{CK})}$ at which the system approaches the two-channel Kondo model fixed point as a function of $J_{0}$ and $\beta$. The numbers shown are the integer parts of the decadic logarithms, $\left|\log _{10}\left(T_{K}^{(2 \mathrm{CK})} / D\right)\right|$. The omitted values for intermediate values of $J_{0}$ correspond to the situation where it is difficult to determine $T_{(2 C K)}$ since the cross-over occurs at relatively high temperature.

a regular strong-couping Kondo fixed point in which the sidecoupled impurity forms a Kondo state with electrons in either left or right channel, depending on which of the two effective exchange constants is larger. The intermediate 2IK fixed point, on the other hand, would be affected only little.

\section{TRIANGULAR TRIPLE QUANTUM DOT}

A triangular triple quantum dot (TQD) model consists of three Anderson-like impurities interconnected by electron hopping and coupled to the conduction bands via hybridization (see also Refs. 92 and 80). The geometry is still that displayed in Fig. 1. Various physical properties of systems with this kind of lattice connectivity have been previously studied $^{52.57}$. In this work we focus on the Hamiltonian

$$
H=H_{\mathrm{b}}+H_{\mathrm{imp}}+H_{\mathrm{c}}
$$

where $H_{\mathrm{b}}$ remains the same as in Eq. (1), while the impurity and coupling Hamiltonians become

$$
\begin{aligned}
H_{\mathrm{imp}} & =\sum_{i}(U / 2)\left(n_{i}-1\right)^{2}+\sum_{i j, \sigma} t_{i j} d_{i \sigma}^{\dagger} d_{j \sigma}+\text { H.c. }, \\
H_{\mathrm{c}} & =V \sum_{k \sigma} c_{L k \sigma}^{\dagger} d_{1 \sigma}+\text { H.c. } \\
& +V \sum_{k \sigma} c_{R k \sigma}^{\dagger} d_{3 \sigma}+\text { H.c. }
\end{aligned}
$$

Here $U$ is the on-site electron-electron repulsion, $t_{i j}$ are hopping matrix elements, $d_{i \sigma}^{\dagger}$ is the spin- $\sigma$ electron creation operator on site $i$, and $n_{i}=\sum_{\sigma} d_{i \sigma}^{\dagger} d_{i \sigma}$ is the occupancy operator on site $i$. Finally, $V$ is the hybridization matrix element which is assumed to be a constant independent of $k$. The hybridization strength is then $\Gamma=\pi \rho V^{2}$ with the conduction band density of states $\rho=1 /(2 D)$. There are no terms of the form $\epsilon_{i} n_{i}$ which are typically included to describe the effect 
of gate voltages. We instead assumed that the occupancy is near half filling, as implied by $(U / 2)\left(n_{i}-1\right)^{2}$ terms. The particle-hole symmetry is broken by any finite hopping which makes the lattice connectivity non-bipartite and it should be noted that the deviation from half-filling may become significant if hopping matrix elements are large, $t_{i j} \sim U$. We are, however, more interested in the limit of small $t_{i j} \ll U$. If the model parameters are chosen so that $J_{i j}=4 t_{i j}^{2} / U$ and $\rho J_{K}=8 \Gamma / \pi U$, models (1) and (11) have namely very similar properties as long as $t_{i j} \ll U$. The essential difference, however, is that in model (11) the electrons are allowed to tunnel from left to right conduction leads. It is known that interchannel charge transfer destabilizes both the two-impurity and the two-channel Kondo-model fixed points ${ }^{74,80}$, therefore we expect that the ground state of the TQD system will be Fermiliquid for any choice of parameters. We parameterize the tunneling matrix elements using a new quantity $\alpha$ as

$$
\begin{aligned}
t_{13} & =t_{0} \sin (\alpha \pi / 2), \\
t_{12}=t_{23} & =t_{0} \cos (\alpha \pi / 2) .
\end{aligned}
$$

Hoppings $t_{i j}$ can be related to exchange constants $J_{i j}$ only in the limit $t_{i j} \ll U$ : then the relation between $\alpha$ and $\beta$ is given by $\sin (\beta \pi / 2) \propto \sin ^{2}(\alpha \pi / 2)$.

The zero-temperature conductance through the TQD can be related to the quasiparticle phase shifts as 22,27,28,103,104,105

$$
G=G_{0} \sin ^{2}\left(\delta_{\mathrm{q} \cdot \mathrm{p} .}^{\text {even }}-\delta_{\mathrm{q} \cdot \mathrm{p} .}^{\text {odd }}\right),
$$

where $G_{0}=2 e^{2} / h$ is the conductance quantum. The phase shifts can be easily extracted from the renormalization flow diagrams when the system reaches the low-temperature stable Fermi-liquid fixed point $27,28,29,73,77,104,105,106,107,108$. Phase shifts may be constrained in the presence of the particle-hole symmetry of certain kinds 67 . For $\alpha=1$, model (11) is particle-hole symmetric, however the quasiparticle scattering phase shifts are not fixed to any particular value; the stable fixed point therefore belongs to the plane P1, see Sec. [III For generic $\alpha$, i.e. $\alpha \neq 1$ and $\alpha \neq 0$, the lattice on which the Hamiltonian (11) is defined is not bipartite, which immediately precludes any kind of particle-hole symmetry; the stable fixed point must thus belong to the plane P2. Finally, the case $\alpha=0$ was discussed in Refs. 28, 80,92: the phase shift in odd channel is constrained to $\pi / 2$, while there is zero phase shift in the even channel (this fixed point also belongs to the plane $\mathrm{P} 2)$.

Properties of the double quantum dot (DQD) systems (i.e. $\alpha=1$ limit) have been studied in a number of works using various methods $74,103,109,110,111,112,113,114,115,116,117$. These calculations show that the conductance of DQD goes to zero in the limit of small $t$ (as the dots become decoupled) as well as in the limit of large $t$ (as the electrons occupy the bonding molecular orbital); the conductance peaks at the unitary conductance limit for $t=t^{*}$ such that $J_{\text {eff }} \sim c T_{K}^{(1)}$ where $J_{\text {eff }}=$ $4 t^{2} / U$. The difference of phase shifts $\Delta=\delta_{\mathrm{q} \cdot \mathrm{p} \text {. }}^{\text {even }}-\delta_{\mathrm{q} \cdot \mathrm{p} \text {. }}^{\text {odd }}$ varies continuously and smoothly from 0 to $\pi$ at $t$ goes from 0 to $\infty$ (it should be noted that scattering phase shifts are defined modulo $\pi$ ). The fact that conductance becomes unitary at some some value of $t$ is a simple consequence of $\Delta$ going through $\pi / 2$; what is less trivial, but not unexpected, is that this occurs when $J_{\text {eff }} \sim c T_{K}^{(1)}$.

The conductance of TQD in series, on the other hand, was found to be unitary for all values of $t$, which is a direct consequence of the phase shift pinning at $\delta_{\mathrm{q} \cdot \mathrm{p} .}^{\text {odd }}=\pi / 2$ and $\delta_{\text {q.p. }}^{\text {even }}=027,28,80,92$.

At zero temperature the variation of the phase shifts as a function of $t_{0}$ and $\alpha$ might be expected to be continuous and smooth throughout the entire parameter plane. Based on the considerations of the variation of phase shifts as a function of $t$ in serial DQD and TQD problems, we can make qualitative predictions about the dependence of the conductance on $t_{0}$ and $\alpha$. For $t_{0} \ll t^{*}$ we expect the conductance to drop monotonously from $G_{0}$ to 0 as $\alpha$ goes from 0 to 1 . For $t_{0} \sim t^{*}$ the conductance is $G_{0}$ for $\alpha=0$ and $\sim G_{0}$ for $\alpha=1$; it is reasonable to assume that the conductance drops to zero for some intermediate value of $\alpha$. Finally, for $t_{0} \gg t^{*}$ we expect both a conductance zero and a conductance peak for some intermediate values of $\alpha$.

The results of a NRG calculation of the "zero-temperature" conductance are shown in Fig. 12, We have taken into account the spin $\mathrm{SU}(2)$ symmetry and parity $\mathrm{Z}_{2}$ symmetry. In these computations, a large value of the discretization parameter has been used, $\Lambda=8$; up to 150 NRG iterations were performed, which corresponds to an extremely low temperature of $T \sim 10^{-68} D$. Nevertheless, in some parameter ranges the stable Fermi-liquid fixed point has not been reached at this temperature. In the shaded box in Fig. 12, the excitation spectra at the last NRG iteration for some values of $t_{0}$ were not those of Fermi liquid fixed points. Furthermore, to the right of the shaded box, in the immediate vicinity of $\alpha=1$, spectra obtained at the last NRG iteration correspond to Fermiliquid fixed point in the P1 plane (see Sec. IIII), while it is known that the system should eventually cross over to a stable Fermi-liquid fixed point in the P2 plane. These findings can be explained by the presence of the exponentially low energy scales when the impurity 2 is nearly decoupled; see Eqs. (7) and (8) for the equivalent behavior in the Kondo-like model. As $\alpha$ goes to 1 , the cross-over temperature scale $T_{\Delta}$ becomes arbitrarily low, thus the "zero-temperature" conductance is experimentally irrelevant.

Transport experiments are decidedly performed at some finite temperature $T_{\text {exp. }}$. As $\alpha$ is increased, at some value $\alpha_{D}$ the crossover temperature $T_{\Delta}$ will suddenly decrease (exponentially) below $T_{\text {exp }}$. For all practical purposes, the quantum dot 2 will then be effectively decoupled from the rest of the system and its local moment will not be screened. It should be emphasized that $\alpha_{D}$ is less than 1; at finite temperature, the local moment effectively decouples from the rest of the system at $\alpha=\alpha_{D}$ even though electrons are still able to hop on the quantum dot.

Interestingly, this behavior also implies that the conductance can be abruptly changed by driving the crossover temperature below the experimental temperature by relatively small variation of the gate voltages that control $\alpha^{18}$. To our knowledge, this type of abrupt conductance change has not yet been experimentally observed.

In Fig. 13 we show the low-temperature conductance 


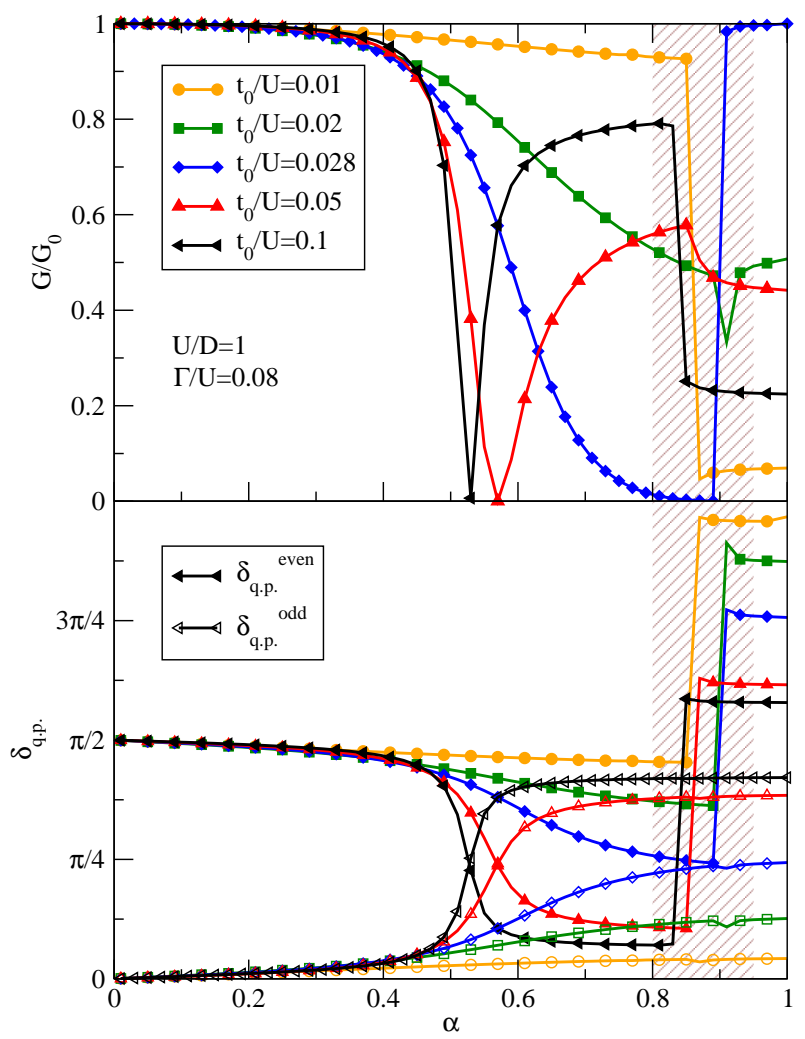

Figure 12: "Zero-temperature" linear conductance through the upper edge of the triangular cluster and the quasiparticle scattering phase shifts as a function of the interdot-tunneling-asymmetry factors $\alpha$ for a range of tunneling parameters $t_{0}$. In the shaded box, the spectrum at the last NRG iteration does not correspond to that of a Fermi-liquid fixed point. The critical $t^{*}$ corresponding to the maximal conduction in the DQD model is approximately $t^{*}=0.028 U$.

through the triangular TQD for a range of $\alpha$ as a function of $t_{0}$. Particularly interesting is how the deviation from the unitary conductance in the linear TQD ( $\alpha=0$ limit) develops when hopping between the first and the third impurity is allowed $(\alpha \neq 0)$. The differences are most significant for large values of $t_{0}$; this coincides with the regime of $t_{i j} \sim U$ where the quantum dots are no longer restrained to half-filling for $\alpha \neq 0$. The change in scattering phase shifts can thus be related to a change in the occupancy (Friedel sum rule). For larger values of $\alpha$, the conductance may become zero at some value of $t_{0}$, see the $\alpha=0.75$ plot in Fig. 13. For $\alpha=1$ we recover the known results for the conductance of the DQD system, but we must keep in mind that for $\alpha \lesssim 1$, these results correspond to a finite temperature, $T_{\exp } \gg T_{\Delta}$.

\section{CONCLUSION}

The very rich phase diagram of the three-impurity twochannel system makes this a very useful toy model to study the possible behavior of generic two-channel quantum impu- rity models. We find all the fixed points familiar from simpler impurity models and we find interesting crossovers, such

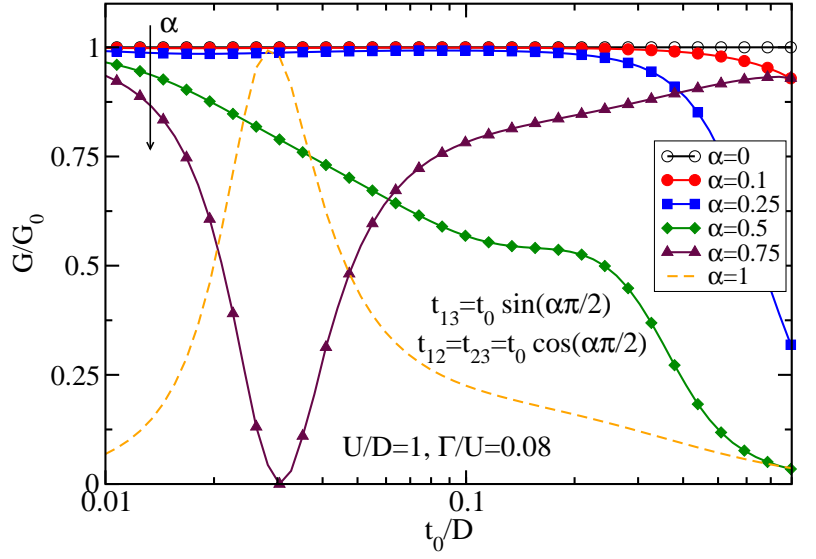

Figure 13: "Zero-temperature" linear conductance through the upper edge of the triangular cluster as a function of the inter-dot tunneling $t_{0}$ for a range of tunneling-asymmetry factors $\alpha$.

as that between the two-impurity and the two-channel Kondo model non-Fermi-liquid fixed points.

In a related triangular triple quantum dot problem we have demonstrated that the presence of energy scales which are extremely (exponentially) low implies that the "zerotemperature" conductance is experimentally irrelevant. In theoretical studies of impurity clusters it is thus imperative to consider the thermal effects and to determine the temperature scale, below which the the transport properties of the system are determined by its ground state.

It would be interesting to extend these studies to threeimpurity three-channel models. Triple quantum dot systems with three conduction leads can be easily manufactured today. In order to determine the transport properties of such nanostructures in all parameter regimes (in particular in the low temperature regime where correlation effects and Kondo physics play a central role), an unbiased method such as NRG is required. NRG becomes numerically highly demanding method in the case of multi-channel problems due to the high degeneracy of quantum states that need to be considered. While two-channel calculations are now performed routinely, only very few three-channel calculations have been reported in the literature so far $23,24,118$. Fortunately, the transport properties at low temperatures (if the system is near a Fermi-liquid fixed point) can be extracted from the energy levels of the NRG eigenvalue flow alone, which requires far less computational resources than calculations of thermodynamic and dynamic (spectral) quantities.

\section{Acknowledgments}

The authors acknowledge support of the SRA under Grant No. P1-0044. 
1 J. Kondo, Prog. Theor. Phys. 32, 37 (1964).

2 G. Grüner and A. Zawadowski, Rep. Prog. Phys. 37, 1497 (1974).

3 A. C. Hewson, The Kondo Problem to Heavy-Fermions (Cambridge University Press, Cambridge, 1993).

${ }^{4}$ L. I. Glazman and M. E. Raikh, JETP Lett. 47, 452 (1988).

${ }^{5}$ L. Kouwenhoven and L. Glazman, Physics World Jan, 33 (2001).

${ }^{6}$ A. Georges, G. Kotliar, W. Krauth, and M. J. Rozenberg, Rev. Mod. Phys. 68, 13 (1996).

7 K. G. Wilson, Rev. Mod. Phys. 47, 773 (1975).

${ }^{8}$ H. R. Krishna-murthy, J. W. Wilkins, and K. G. Wilson, Phys. Rev. Lett. 35, 1101 (1975).

${ }^{9}$ M. Yoshida, M. A. Whitaker, and L. N. Oliveira, Phys. Rev. B 41, 9403 (1990).

10 W. C. Oliveira and L. N. Oliveira, Phys. Rev. B 49, 11986 (1994).

11 K. Chen and C. Jayaprakash, Phys. Rev. B 52, 14436 (1995).

12 H. R. Krishna-murthy, J. W. Wilkins, and K. G. Wilson, Phys. Rev. B 21, 1003 (1980).

${ }^{13}$ R. Bulla, A. C. Hewson, and T. Pruschke, J. Phys.: Condens. Matter 10, 8365 (1998).

${ }^{14}$ W. Hofstetter, Phys. Rev. Lett. 85, 1508 (2000).

15 F. B. Anders and A. Schiller, Phys. Rev. Lett. 95, 196801 (2005).

16 R. Peters, T. Pruschke, and F. B. Anders, Phys. Rev. B 74, 245114 (2006).

17 A. Weichselbaum and J. von Delft, Phys. Rev. Lett. 99, 076402 (2007).

18 R. Žitko and J. Bonča, Phys. Rev. B 73, 035332 (2006).

19 A. I. Tóth and G. Zaránd, Dynamical correlations in the spin-half two-channel kondo model, arxiv:0801.4272 (2008).

${ }^{20}$ R. Bulla, T. Costi, and T. Pruschke, Rev. Mod. Phys. 80, 395 (2008).

21 R. Žitko and J. Bonča, Phys. Rev. B 74, 045312 (2006).

${ }^{22}$ R. Žitko and J. Bonča, Phys. Rev. B 76, 241305(R) (2007).

${ }^{23}$ B. C. Paul and K. Ingersent, Frustration-induced non-fermiliquid behavior in a three-impurity kondo model, condmat/9607190 (1996).

${ }^{24}$ M. Ferrero, L. de Leo, P. Lecheminant, and M. Fabrizio, J. Phys.: Condens. Matter 19, 433201 (2007).

25 R. Žitko and J. Bonča, Phys. Rev. B 74, 224411 (2006).

26 W.-z. Wang, Phys. Rev. B 76, 115114 (2007).

27 A. Oguri, Y. Nisikawa, and A. C. Hewson, J. Phys. Soc. Japan 74, 2554 (2005).

28 A. Oguri and A. C. Hewson, J. Phys. Soc. Japan 74, 988 (2005).

29 Y. Nisikawa and A. Oguri, Phys. Rev. B 73, 125108 (2006).

30 P. Nozières and A. Blandin, J. Physique 41, 193 (1980).

31 C. M. Varma, Z. Nussinov, and W. van Saarloos, Phys. Rep. 361, 267 (2002).

${ }^{32}$ I. Affleck, J. Phys. Soc. Japan 74, 59 (2005).

33 J. Ye, Phys. Rev. B 56, 1316 (1997).

34 J. M. Maldacena and A. W. W. Ludwig, Nucl. Phys. B 506, 565 (1997).

35 J. Ye, Phys. Rev. Lett. 77, 3224 (1996).

36 J. Ye, Phys. Rev. Lett. 79, 1385 (1997).

37 A. M. Sengupta and A. Georges, Phys. Rev. B 49, 10020(R) (1994).

38 J. Ye, Phys. Rev. B 56, R489 (1997).

39 D. L. Cox and M. Jarrell, J. Phys.: Condens. Matter 8, 9825 (1996).

40 D. L. Cox and A. Zawadowski, Adv. Phys. 47, 599 (1998).

${ }^{41}$ R. M. Potok, I. G. Rau, H. Shtrikman, Y. Oreg, and D. GoldhaberGordon, Nature 446, 167 (2007).

${ }^{42}$ A. Vidan, R. M. Westervelt, M. Stopa, M. Hanson, and A. C.
Gossard, Appl. Phys. Lett. 85, 3602 (2004).

${ }^{43}$ L. Gaudreau, S. A. Studenikin, A. S. Sachrajda, P. Zawadzki, A. Kam, J. Lapointe, M. Korkusinski, and P. Hawrylak, Phys. Rev. Lett. 97, 036807 (2006).

${ }^{44}$ F. R. Waugh, M. J. Berry, D. J. Mar, R. M. Westervelt, K. L. Campman, and A. C. Gossard, Phys. Rev. Lett. 75, 705 (1995).

45 M. Korkusinski, I. P. Gimenez, P. Hawrylak, L. Gaudreau, S. A. Studenikin, and A. S. Sachrajda, Phys. Rev. B 75, 115301 (2007).

${ }^{46}$ D. Schröer, A. D. Greentree, L. Gaudreau, K. Eberl, L. C. L. Hollenberg, J. P. Kotthaus, and S. Ludwig, Phys. Rev. B 76, 075306 (2007).

47 T. Jamneala, V. Madhavan, and M. F. Crommie, Phys. Rev. Lett. 87, 256804 (2001).

48 C. A. Stafford and S. Das Sarma, Phys. Rev. Lett. 72, 3590 (1994).

49 G. Chen, G. Klimeck, S. Datta, G. Chen, and W. A. Goddard, Phys. Rev. B 50, 8035 (1994).

50 T. Kuzmenko, K. Kikoin, and Y. Avishai, Europhys. Lett. 64, 218 (2003).

51 G. Zaránd, A. Brataas, and D. Goldhaber-Gordon, Solid State Comm. 126, 463 (2003).

52 T. Kuzmenko, K. Kikoin, and Y. Avishai, Phys. Rev. Lett. 96, 046601 (2006).

53 A. Oguri, Y. Nisikawa, and Y. Tanaka, Kondo screening of a high-spin nagaoka state in a triangular quantum dot, condmat/0606313 (2006).

${ }^{54}$ M. E. Torio, K. Hallberg, and C. R. Proetto, Europhys. Lett. 73, 239 (2006).

55 Z.-t. Jiang, Q.-f. Sun, and Y. Wang, Phys. Rev. B 72, 045332 (2005).

56 M. L. Ladrón de Guevara and P. A. Orellana, Phys. Rev. B 73, 205303 (2006).

57 Z.-T. Jiang and Q. feng Sun, J. Phys.: Condens. Matter 19, 156213 (2007).

58 K. Kikoin, Low. temp. phys. 33, 153 (2007).

59 A. M. Lobos and A. A. Aligia, Phys. Rev. B 74, 165417 (2006).

60 Y. B. Kudasov and V. M. Uzdin, Phys. Rev. Lett. 89, 276802 (2002).

${ }^{61}$ V. V. Savkin, A. N. Rubtsov, M. I. Katsnelson, and A. I. Lichtenstein, Phys. Rev. Lett. 94, 026402 (2005).

62 B. Lazarovits, P. Simon, G. Zaránd, and L. Szunyogh, Phys. Rev. Lett. 95, 077202 (2005).

${ }^{63}$ K. Ingersent, A. W. W. Ludwig, and I. Affleck, Phys. Rev. Lett. 95, 257204 (2005).

64 A. A. Aligia, Phys. Rev. Lett. 96, 096804 (2006).

${ }^{65}$ C. A. Büsser, A. Moreo, and E. Dagotto, Phys. Rev. B 70, 035402 (2004).

66 T. Tanamoto and Y. Nishi, Phys. Rev. B 76, 155319 (2007).

${ }^{67}$ I. Affleck, A. W. W. Ludwig, and B. A. Jones, Phys. Rev. B 52, 9528 (1995).

${ }^{68}$ C. Jayaprakash, H. R. Krishna-murthy, and J. W. Wilkins, Phys. Rev. Lett. 47, 737 (1981).

69 B. A. Jones and C. M. Varma, Phys. Rev. Lett. 58, 843 (1987).

70 B. A. Jones, C. M. Varma, and J. W. Wilkins, Phys. Rev. Lett. 61, 125 (1988).

71 B. A. Jones, B. G. Kotliar, and A. J. Millis, Phys. Rev. B 39, 3415 (1989).

72 B. A. Jones and C. M. Varma, Phys. Rev. B 40, 324 (1989).

73 I. Affleck and A. W. W. Ludwig, Phys. Rev. Lett. 68, 1046 (1992).

${ }^{74}$ G. Zaránd, C.-H. Chung, P. Simon, and M. Vojta, Phys. Rev. Lett. 97, 166802 (2006). 
75 O. Sakai and Y. Shimizu, J. Phys. Soc. Japan 61, 2333 (1992).

76 V. L. Campo and L. N. Oliveira, Phys. Rev. B 70, 153401 (2004).

77 L. Zhu and C. M. Varma, Coherence in the two kondo impurity problem, cond-mat/0607426 (2006).

78 M. Vojta, Rep. Prog. Phys. 66, 2069 (2003).

${ }^{79}$ M. Vojta, Phil. Mag. 86, 1807 (2006).

${ }^{80}$ R. Žitko and J. Bonča, Phys. Rev. Lett. 98, 047203 (2007).

${ }^{81}$ I. Affleck, A. W. W. Ludwig, H.-B. Pang, and D. L. Cox, Phys. Rev. B 45, 7918 (1992).

${ }^{82}$ H. B. Pang and D. L. Cox, Phys. Rev. B 44, 9454 (1991).

${ }^{83}$ H. Pang, Phys. Rev. Lett. 73, 2736 (1994).

84 A. W. W. Ludwig and I. Affleck, Phys. Rev. Lett. 67, 3160 (1991).

85 A. M. Sengupta and Y. B. Kim, Phys. Rev. B 54, 14918 (1996).

86 J. von Delft, G. Zaránd, and M. Fabrizio, Phys. Rev. Lett. 81, 196 (1998).

87 G. Zaránd, T. Costi, A. Jerez, and N. Andrei, Phys. Rev. B 65, 134416 (2002).

${ }^{88}$ I. Affleck and A. W. W. Ludwig, Nucl. Phys. B 360, 641 (1991).

${ }^{89}$ P. Coleman and A. J. Schofield, Phys. Rev. Lett. 75, 2184 (1995).

90 P. Coleman, L. B. Ioffe, and A. M. Tsvelik, Phys. Rev. B 52, 6611 (1995).

91 N. Andrei and A. Jerez, Phys. Rev. Lett. 74, 4507 (1995).

92 R. Žitko, J. Bonča, A. Ramšak, and T. Rejec, Phys. Rev. B 73, 153307 (2006).

93 C. Kolf and J. Kroha, Phys. Rev. B 75, 045129 (2007).

94 H. Kusunose, K. Miyake, Y. Shimizu, and O. Sakai, Phys. Rev. Lett. 76, 271 (1996).

95 M. Pustilnik, L. Borda, L. I. Glazman, and J. von Delft, Phys. Rev. B 69, 115316 (2004).

96 R. Zitko, Nrg ljubljana, http://nrgljubljana.ijs.si/ (2006).

97 H. R. Krishna-murthy, J. W. Wilkins, and K. G. Wilson, Phys. Rev. B 21, 1044 (1980).

98 J. B. Silva, W. L. C. Lima, W. C. Oliveira, J. L. N. Mello, L. N.
Oliveira, and J. W. Wilkins, Phys. Rev. Lett. 76, 275 (1996).

99 V. L. Campo and L. N. Oliveira, Phys. Rev. B 72, 104432 (2005).

100 J. Gan, Phys. Rev. B 51, 8287 (1995).

101 P. S. Cornaglia and D. R. Grempel, Phys. Rev. B 71, 075305 (2005).

102 M. Vojta, R. Bulla, and W. Hofstetter, Phys. Rev. B 65, 140405(R) (2002).

103 A. Georges and Y. Meir, Phys. Rev. Lett. 82, 3508 (1999).

104 M. Pustilnik and L. I. Glazman, Phys. Rev. Lett. 87, 216601 (2001).

105 W. Hofstetter and G. Zarand, Phys. Rev. B 69, 235301 (2004).

106 L. N. Oliveira and J. W. Wilkins, Phys. Rev. B 24, 4863 (1981).

107 L. Borda, G. Zaránd, W. Hofstetter, B. I. Halperin, and J. von Delft, Phys. Rev. Lett. 90, 026602 (2003).

108 P. Mehta, N. Andrei, P. Coleman, L. Borda, and G. Zarand, Phys. Rev. B 72, 014430 (2005)

109 T. Pohjola, J. Konig, M. M. Salomaa, J. Schmid, H. Schoeller, and G. Schon, Europhys. Lett. 40, 189 (1997).

110 T. Aono, M. Eto, and K. Kawamura, J. Phys. Soc. Japan 67, 1860 (1998).

111 W. Izumida and O. Sakai, Phys. Rev. B 62, 10260 (2000).

112 D. Boese, W. Hofstetter, and H. Schoeller, Phys. Rev. B 66, 125315 (2002).

113 R. López, R. Aguado, and G. Platero, Phys. Rev. Lett. 89, 136802 (2002).

114 R. Aguado and D. C. Langreth, Phys. Rev. B 67, 245307 (2003).

115 W. Izumida and O. Sakai, J. Phys. Soc. Japan 74, 103 (2005).

116 C. Karrasch, T. Enss, and V. Meden, Phys. Rev. B 73, 235337 (2006).

117 J. Mravlje, A. Ramšak, and T. Rejec, Phys. Rev. B 73, 241305(R) (2006).

118 L. De Leo and M. Fabrizio, Phys. Rev. Lett. 94, 236401 (2005). 